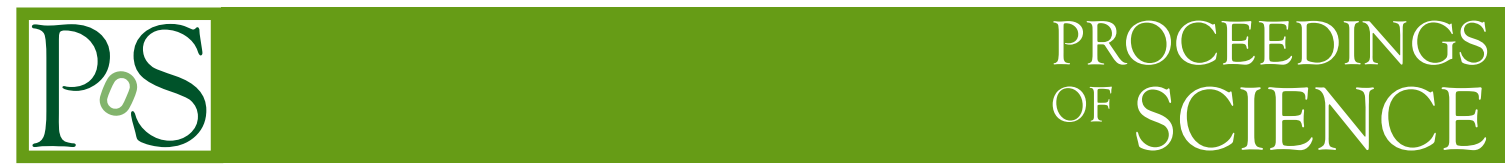

\title{
Radiative and Electroweak Penguin $B$ decays at Belle
}

Yutaro Sato*i

Nagoya University

E-mail: yutaro@hepl.phys.nagoya-u.ac.jp

We report results of radiative and electroweak penguin $B$ decays based on a data sample containing $772 \times 10^{6} B \bar{B}$ pairs recorded by the Belle detector at the KEKB $e^{+} e^{-}$collider. The lepton forwardbackward asymmetry $\mathscr{A}_{\mathrm{FB}}$ for the electroweak penguin process $B \rightarrow X_{S} \ell^{+} \ell^{-}$has been measured for the first time, where $\ell$ is an electron or a muon and $X_{s}$ is a hadronic recoil system with an $s$ quark. In addition, the $B^{0} \rightarrow p \bar{\Lambda} \pi^{-} \gamma$ decay has been searched.

The European Physical Society Conference on High Energy Physics -EPS-HEP2013

18-24 July 2013

Stockholm, Sweden

* Speaker.

${ }^{\dagger}$ A footnote may follow. 


\section{Introduction}

In the standard model (SM), flavor-changing neutral current such as $b \rightarrow s \gamma$ and $b \rightarrow s \ell^{+} \ell^{-}$ decays are forbidden at the tree level and allowed at higher order via the electroweak penguin or $W^{+} W^{-}$box diagrams. Non-SM particles may contribute to the loop and change their decay rate and angular distributions. Therefore, the $b \rightarrow s \gamma$ and $b \rightarrow s \ell^{+} \ell^{-}$decays are good probes to search for new physics. Here, we report first measurement of the lepton forward-backward asymmetry in the $B \rightarrow X_{S} \ell^{+} \ell^{-}$decays and the result of search for $B^{0} \rightarrow p \bar{\Lambda} \pi^{-} \gamma$ decays. Both result is based on a data sample containing $772 \times 10^{6} B \bar{B}$ pairs recorded by the Belle detector at the KEKB $e^{+} e^{-}$ collider.

\section{Lepton forward-backward asymmetry in inclusive $B \rightarrow X_{s} \ell^{+} \ell^{-}$}

Inclusive measurements of $b \rightarrow s \gamma$ and $b \rightarrow s \ell^{+} \ell^{-}$are preferable to exclusive measurements because of lower theoretical uncertainties, although they are experimentally more challenging. The branching fraction of inclusive $B \rightarrow X_{s} \gamma$ and $B \rightarrow X_{s} \ell^{+} \ell^{-}$has been measured by Belle [1], BABAR [3, 4], and CLEO [5]. All of them are consistent with the theoretical SM prediction [6, 7]. The lepton forward-backward asymmetry $\mathscr{A}_{\mathrm{FB}}$ in the $B \rightarrow X_{s} \ell^{+} \ell^{-}$decays defined as

$$
\mathscr{A}_{\mathrm{FB}} \equiv \frac{\Gamma\left(b \rightarrow s \ell^{+} \ell^{-} ; \cos \theta>0\right)-\Gamma\left(b \rightarrow s \ell^{+} \ell^{-} ; \cos \theta<0\right)}{\Gamma\left(b \rightarrow s \ell^{+} \ell^{-} ; \cos \theta>0\right)+\Gamma\left(b \rightarrow s \ell^{+} \ell^{-} ; \cos \theta<0\right)},
$$

is considered to have different and better sensitivity to physics beyond the SM than the branching fraction [8]. Here, $\theta$ is the angle between the $\ell^{+}\left(\ell^{-}\right)$and the $B$ meson three-momenta in the $\ell^{+} \ell^{-}$ center-of-mass (c.m.) frame in $\bar{B}^{0}$ or $B^{-}\left(B^{0}\right.$ or $\left.B^{+}\right)$decays.

The $B \rightarrow X_{s} \ell^{+} \ell^{-}$decays are reconstructed from dilepton pair $\ell^{+} \ell^{-}\left(e^{+} e^{-}\right.$or $\left.\mu^{+} \mu^{-}\right)$and one of ten reconstructed hadronic states: $K^{-}, K^{-} \pi^{+}, K^{-} \pi^{0}, K_{S}^{0} \pi^{-}, K^{-} \pi^{+} \pi^{0}, K^{-} \pi^{+} \pi^{-}, K_{S}^{0} \pi^{-} \pi^{0}$, $K^{-} \pi^{+} \pi^{-} \pi^{+}, K^{-} \pi^{+} \pi^{-} \pi^{0}$, and $K_{S}^{0} \pi^{-} \pi^{+} \pi^{-}$. To examine the dilepton mass squared $q^{2}$ dependence of $\mathscr{A}_{\mathrm{FB}}$, we divide the data into 4 bins of measured $q^{2}$ : [0.2, 4.3], [4.3, 7.3(8.1)], [10.5(10.2), $11.8(12.5)],[14.3,25.0] \mathrm{GeV}^{2} / c^{4}$ for the electron (muon) channel, where the gap regions correspond to the veto regions for charmonium background events. In order to extract $\mathscr{A}_{\mathrm{FB}}$, an extended unbinned maximum likelihood fit is performed for each $q^{2}$ bin simultaneously fitting the four $M_{\mathrm{bc}}$ distributions: events with $\cos \theta>0$ for electron/muon channel and event with $\cos \theta<0$ for electron/muon channel. The likelihood function consists of four components: signal, self cross-feed, peaking background, and combinatorial background, where we consider three peaking background sources: charmonium background from $B \rightarrow J / \psi(\psi(2 S)) X_{s}$ decays with $J / \psi(\psi(2 S)) \rightarrow \ell^{+} \ell^{-}$, $B \rightarrow D^{(*)} n \pi(n>0)$ decays with misidentification of two charged pions as two leptons, and $B \rightarrow J / \psi(\psi(2 S)) X_{s}$ decays with swapped misidentification of a lepton and a pion. Figure 1 shows the $M_{\mathrm{bc}}$ distributions for $B \rightarrow X_{s} e^{+} e^{-}$and $B \rightarrow X_{s} \mu^{+} \mu^{-}$candidates with positive and negative $\cos \theta$. The total signal yields for $B \rightarrow X_{s} e^{+} e^{-}$and $B \rightarrow X_{s} \mu^{+} \mu^{-}$are measured to be $139.8 \pm$ 18.6 (stat) and $160.8 \pm 20.0$ (stat), respectively. Figure 2 shows the $\mathscr{A}_{\mathrm{FB}}$ distribution as a function of $q^{2}$. In the high $q^{2}$ bins, the results are consistent with the SM prediction. In the bins above the $J / \psi$ veto region, $\mathscr{A}_{\mathrm{FB}}<0$ is excluded at the $2.3 \sigma$ level, where $\sigma$ is the standard deviation. In the lowest $q^{2}$ bin $\left(q^{2}<4.3 \mathrm{GeV}^{2} / c^{4}\right)$, the result is within $1.8 \sigma$ of the SM expectation. 

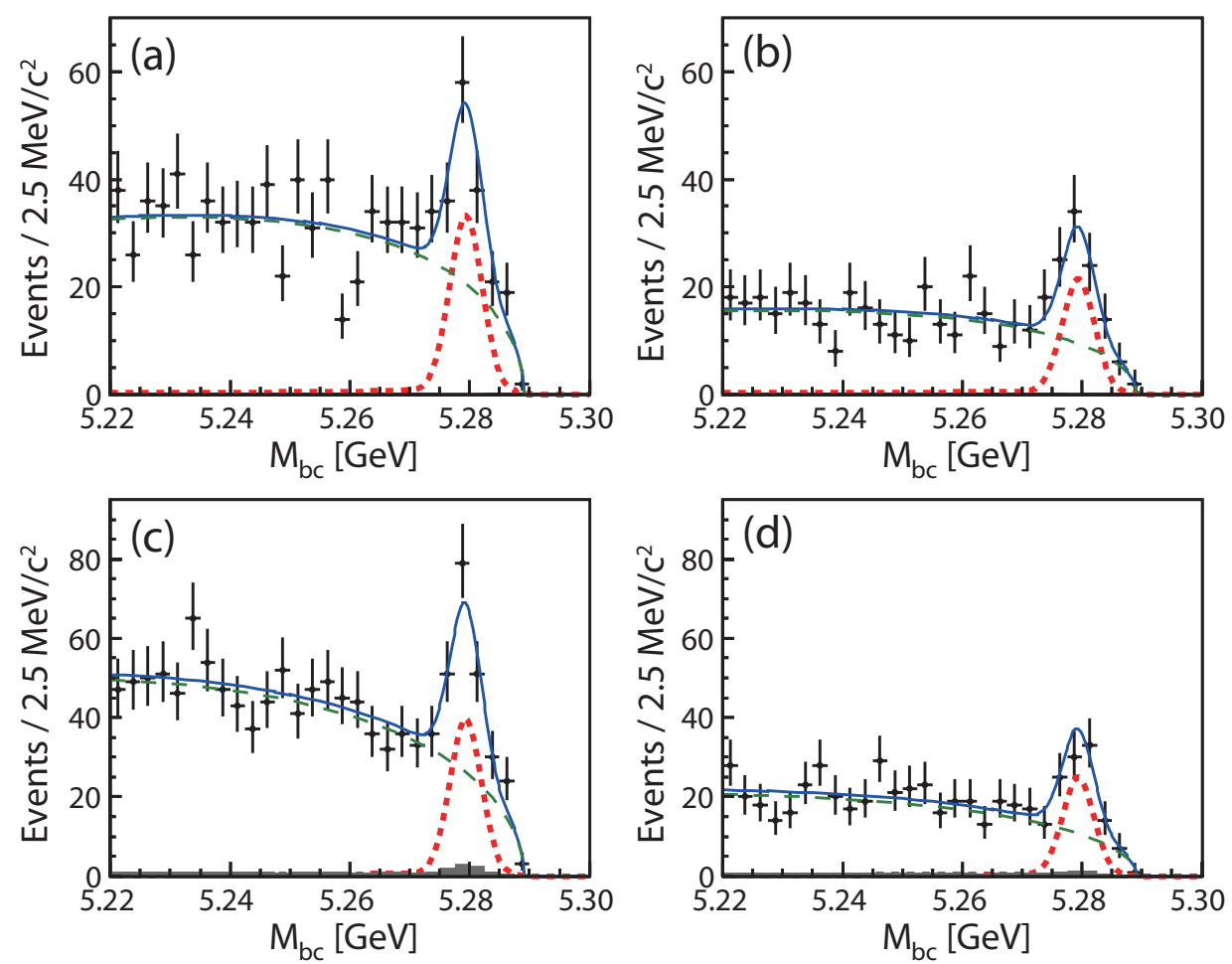

Figure 1: $M_{\mathrm{bc}}$ distributions for (a) $B \rightarrow X_{s} e^{+} e^{-}$candidates with $\cos \theta>0$, (b) $B \rightarrow X_{s} e^{+} e^{-}$candidates with $\cos \theta<0$, (c) $B \rightarrow X_{s} \mu^{+} \mu^{-}$candidates with $\cos \theta>0$, and (d) $B \rightarrow X_{s} \mu^{+} \mu^{-}$candidates with $\cos \theta<0$. The thicker dashed curve (red) shows the sum of the signal and the self cross-feed components. The thinner dashed curve (green) shows the combinatorial background component. The filled histogram (gray) shows the peaking background component. The sums of all components are shown by the solid curve (blue).

\section{Search for $B^{0} \rightarrow p \bar{\Lambda} \pi^{-} \gamma$}

In the Monte Carlo (MC) simulation of the $s \rightarrow X_{s}$ fragmentation/hadronization process by JETSET [9], the $X_{s}$ with a $\Lambda$ in the final state contribute only at $1 \%$ level. This is consistent with the known baryonic $B$ decay rate, $\mathscr{B}\left(B^{+} \rightarrow p \bar{\Lambda} \gamma\right)=\left(2.45_{-0.38}^{+0.44} \pm 0.22\right) \times 10^{-6}[10]$. There is an intriguing feature of this three-body decay that the mass of the $p \Lambda$ system is peaked near threshold. A similar feature is also seen in many other baryonic $B$ decay processes and quite likely offers the explanation to the observed hierarchy, e.g. $\mathscr{B}\left(B^{+} \rightarrow p \bar{\Lambda} \pi^{+} \pi^{-}\right)>\mathscr{B}\left(B^{0} \rightarrow p \bar{\Lambda} \pi^{-}\right)>\mathscr{B}\left(B^{+} \rightarrow p \bar{\Lambda}\right)$ and $\mathscr{B}\left(B^{0} \rightarrow p \bar{\Lambda}_{c}^{-} \pi^{+} \pi^{-}\right)>\mathscr{B}\left(B^{+} \rightarrow p \bar{\Lambda}_{c}^{-} \pi^{+}\right)>\mathscr{B}\left(B^{0} \rightarrow p \bar{\Lambda}_{c}^{-}\right) \llbracket 11$.

The signal yield of the $B^{0} \rightarrow p \bar{\Lambda} \pi^{-} \gamma$ mode is extracted from a two-dimensional extended unbinned maximum likelihood fit. The fit result is shown in Fig 3 The fitted signal yield is $9.5_{-10.7}^{+11.5}$ with a statistical significance of 0.9 . The statistical significance is defined as $\sqrt{-2 \ln \left(L_{0} / L_{\max }\right)}$, where $L_{0}$ and $L_{\max }$ are the likelihood values obtained by the fit with and without the signal yield fixed to zero, respectively. The branching fraction $\mathscr{B}$ is calculated using the following formula:

$$
\mathscr{B}=\frac{N_{\text {sig }}}{\varepsilon \cdot N_{B \bar{B}}},
$$




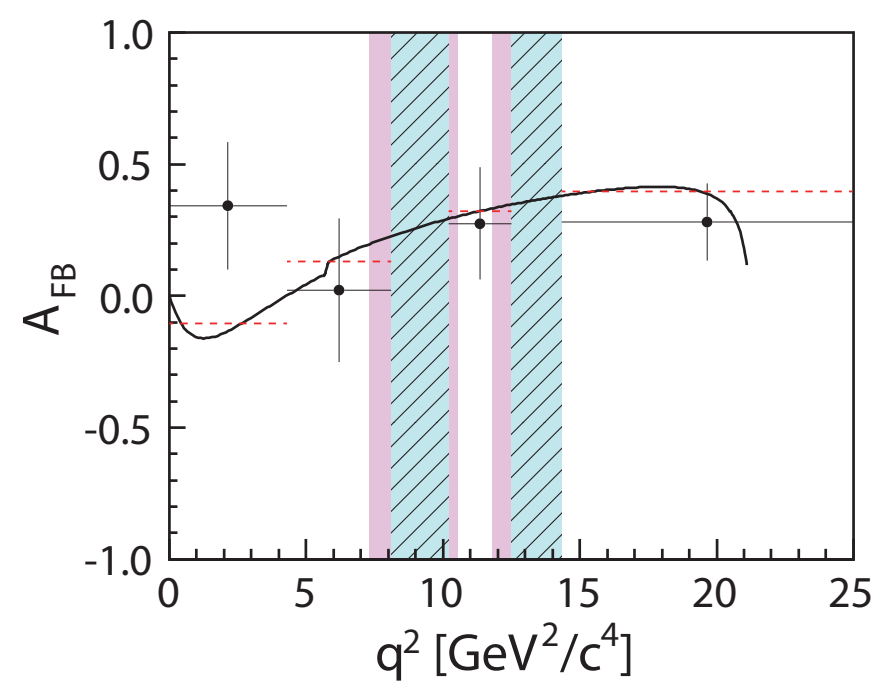

Figure 2: $\mathscr{A}_{\mathrm{FB}}$ for each $q^{2}$ bin. The curve (black) and horizontal dashed line (red) represent the SM prediction while filled circles show the fit results. The $J / \psi$ and $\psi(2 \mathrm{~S})$ veto regions are shown as blue hatched regions. For the electron channel, red shaded regions are added to the veto regions due to the large bremsstrahlung effect.

where $N_{\text {sig }}, N_{B \bar{B}}$, and $\varepsilon$ are the fitted signal yield, the number of $B \bar{B}$ pairs, and the reconstruction efficiency of signal, respectively. The reconstruction efficiency for the signal is estimated to be $5.27 \%$ from the MC samples and control data sample. Since the observed yield for $B^{0} \rightarrow p \bar{\Lambda} \pi^{-} \gamma$ is not significant enough, we evaluate the $90 \%$ confidence level Bayesian upper limit $\left(\mathscr{B}_{U L}\right)$ to be $6.5 \times 10^{-7}$. We conclude that the decay under study does not follow the expected hierarchy, namely $\mathscr{B}\left(B^{0} \rightarrow p \bar{\Lambda} \pi^{-} \gamma\right)>\mathscr{B}\left(B^{+} \rightarrow p \bar{\Lambda} \gamma\right)$.

\section{Summary}

We present new results on radiative and electroweak penguin $B$ decays obtained from a data sample containing $772 \times 10^{6} B \bar{B}$ pairs recorded by the Belle detector at the KEKB $e^{+} e^{-}$collider. The lepton forward-backward asymmetry $\mathscr{A}_{\mathrm{FB}}$ for the electroweak penguin process $B \rightarrow X_{S} \ell^{+} \ell^{-}$ has been measured for the first time. For $q^{2}>10.2 \mathrm{GeV}^{2} / c^{4}, \mathscr{A}_{\mathrm{FB}}<0$ is excluded at the $2.3 \sigma$ level. For $q^{2}<4.3 \mathrm{GeV}^{2} / c^{4}$, the result is within $1.8 \sigma$ of the SM expectation. The results can be used to constrain extensions of the SM. The $B^{0} \rightarrow p \bar{\Lambda} \pi^{-} \gamma$ decay has been searched and set the $90 \%$ confidence level Bayesian upper limit $\left(\mathscr{B}_{U L}\right)$ to be $6.5 \times 10^{-7}$. We conclude that the decay under study does not follow the expected hierarchy, namely $\mathscr{B}\left(B^{0} \rightarrow p \bar{\Lambda} \pi^{-} \gamma\right)>\mathscr{B}\left(B^{+} \rightarrow p \bar{\Lambda} \gamma\right)$.

\section{References}

[1] A. Limosani et al. (Belle Collaboration), Phys. Rev. Lett. 103, 241801 (2009).

[2] M. Iwasaki et al. (Belle Collaboration), Phys. Rev. D72, 092005 (2005).

[3] J. P. Lees et al., (BABAR Collaboration), Phys. Rev. D86, 11208 (2012). 

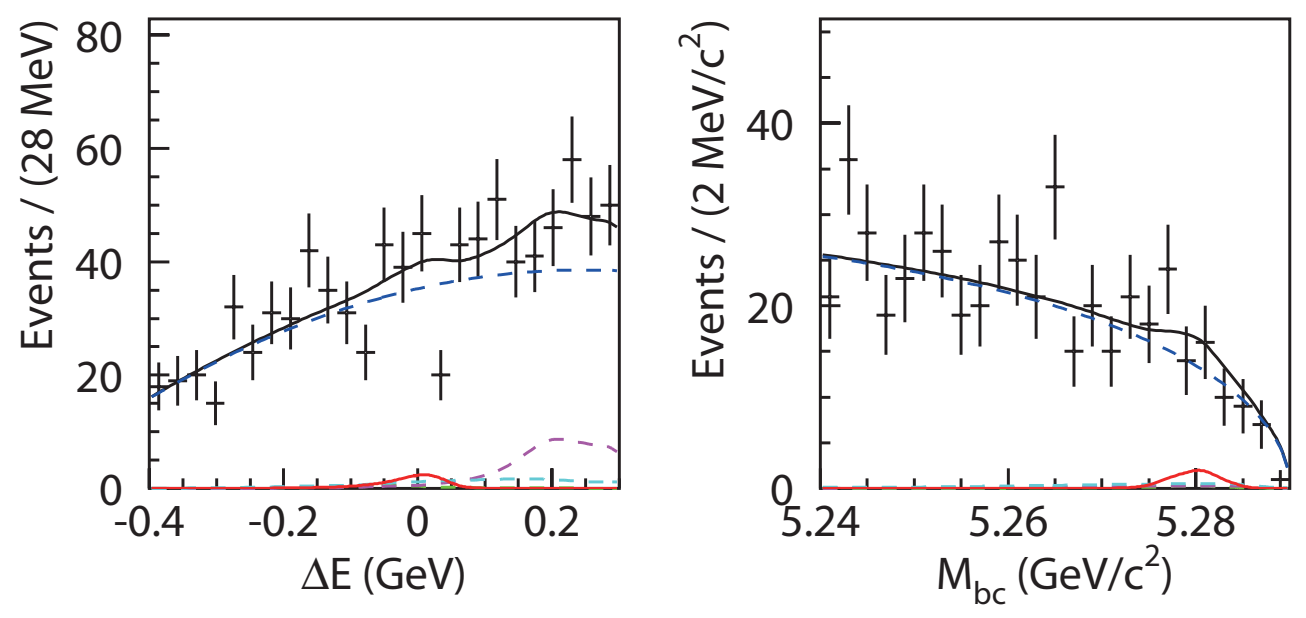

Figure 3: $M_{\mathrm{bc}}$ and $\Delta E$ distribution. The solid (red) curve shows the signal component. The dashed (green) curve shows the self-cross feed component. The (blue) curve shows the continuum background component. The dashed (magenta and cyan) lines show $B \rightarrow p \Lambda \gamma$ and $p \Lambda \pi^{0}$ background events. The solid (black) line shows the sum of all components.

[4] B. Aubert et al. (BABAR Collaboration), Phys. Rev. Lett. 93, 081802 (2004).

[5] S. Chen et al. (CLEO Collaboation), Phys. Rev. Lett. 87, 251807 (2002).

[6] M. Misiak et al., Phys. Rev. Lett. 98, 022002 (2007).

[7] A. Ali, E. Lunghi, C. Greub, and G. Hiller, Phys. Rev. D66, 034002 (2002).

[8] S. Fukae, C.S. Kim, T. Morozumi, and T. Yoshikawa, Phys. Rev. D59, 074013 (1999).

[9] T. Sjostrand, hep-ph/9508391.

[10] M.-Z. Wang et al., (Belle Collaboration), Phys. Rev. D76, 052004 (2007).

[11] J. Beringer et al., (Particle Data Group), Phys. Rev. D86, 010001 (2012). 